\title{
Comparison of Electrocautery Platforms for Pulse Generator Replacement Procedures
}

\author{
Caleb Chiang ${ }^{1}$, Muhammad Ayub ${ }^{1}$, Kousik Krishnan ${ }^{1}$, Richard Trohman ${ }^{1}$, Parikshit \\ Sharma $^{1}$, Timothy Larsen ${ }^{1}$, Henry Huang ${ }^{1}$, Janet Haw $^{1}$, and Jeremiah Wasserlauf ${ }^{1}$ \\ ${ }^{1}$ Rush University Medical Center
}

July 25, 2021

\begin{abstract}
Introduction: Lead damage can occur during cardiovascular implantable electronic device (CIED) generator replacement procedures, resulting in additional procedures and added costs. PlasmaBlade (PL) and PhotonBlade (PH) are two commercially available insulated electrocautery devices designed to reduce this risk. Objective: This study compared complication rates of PL and PH in CIED generator replacement procedures. Methods: This retrospective study analyzed transvenous CIED generator replacements using PL or PH from 2017-2020. The primary endpoint was lead damage. Secondary endpoints included rate of pocket hematoma, superficial infection, and infections requiring device removal. Results: A total of 209 patients were included for analysis, including 86 procedures (188 leads) using PL and 123 procedures (266 leads) using PH. Lead insulation material consisted of $237(52.2 \%)$ silicone, $66(14.5 \%)$ polyurethane, and $151(33.3 \%)$ copolymer leads, with no significant difference in frequency of insulation materials $(\mathrm{p}=0.91)$. The rate of lead damage was low for both $\mathrm{PL}$ and $\mathrm{PH}(0 \mathrm{vs} .0 .4 \%, \mathrm{p}=0.59)$. There was no significant difference in pocket hematoma ( $1.2 \%$ vs. $1.6 \%, \mathrm{p}=0.43)$, superficial infection $(1.2 \%$ vs. $0.8 \%, \mathrm{p}=0.49)$, or infection requiring device removal $(1.2 \%$ vs. $0 \%, \mathrm{p}=0.41)$. The mean absolute change in lead impedance from pre- to post-procedure was similar between PL and PH $(72.3 \pm 126.1$ ohms vs. $60.6 \pm 72.3 \mathrm{ohms}, \mathrm{p}=0.10)$. Conclusions: There was a low rate of complications after CIED replacement procedures, with no significant difference between the PL and PH groups. A majority of leads with silicone insulation material may have contributed to the low rate of lead damage.
\end{abstract}

\section{Hosted file}

JCE Submission 7.15.docx available at https://authorea.com/users/427429/articles/531612comparison-of-electrocautery-platforms-for-pulse-generator-replacement-procedures 